\title{
Characterization of the CALIBAN Critical Assembly Neutron Spectra using Several Adjustment Methods Based on Activation Foils Measurement
}

\author{
Pierre Casoli ${ }^{1, a}$, Gilles Grégoire ${ }^{2}$, Guillaume Rousseau ${ }^{1}$, Xavier Jacquet ${ }^{1}$, and Nicolas Authier ${ }^{1}$ \\ ${ }^{1}$ CEA, Centre de Valduc, 21120 Is-sur-Tille, France \\ ${ }^{2}$ CEA, DEN, DER, Dosimetry, Sensors Instrumentation and Laboratory, Cadarache, \\ 13108 Saint Paul lez Durance, France
}

\begin{abstract}
CALIBAN is a metallic critical assembly managed by the Criticality, Neutron Science and Measurement Department located on the French CEA Center of Valduc. The reactor is extensively used for benchmark experiments dedicated to the evaluation of nuclear data, for electronic hardening or to study the effect of the neutrons on various materials. Therefore CALIBAN irradiation characteristics and especially its central cavity neutron spectrum have to be very accurately evaluated. In order to strengthen our knowledge of this spectrum, several adjustment methods based on activation foils measurements are being studied for a few years in the laboratory. Firstly two codes included in the UMG package have been tested and compared: MAXED and GRAVEL. More recently, the CALIBAN cavity spectrum has been studied using CALMAR, a new adjustment tool currently under development at the CEA Center of Cadarache. The article will discuss and compare the results and the quality of spectrum rebuilding obtained with the UMG codes and with the CALMAR software, from a set of activation measurements carried out in the CALIBAN irradiation cavity.
\end{abstract}

\section{Introduction}

The Criticality, Neutron Science and Measurement Department located on the French CEA Research Center of Valduc operates several experimental facilities mainly devoted to the production of neutron and gamma mixed fluxes. Two of these facilities, the metallic core reactors CALIBAN and PROSPERO are currently unique in Europe. Initially designed for electronic hardening studies, they are now used for different types of studies, from nuclear data evaluation to radiation shielding. To allow the analysis of these experiments, neutron spectra of the reactors have been studied since the beginning of their operation in the 70's, combining simulation calculations and activations measurements.

In 2008, a benchmark of CALIBAN was published [1], providing a highly accurate description of the reactor. This work allowed the enhancement of the quality of the reactor simulation calculations and was used to test new spectrum adjustment techniques and tools.

\footnotetext{
${ }^{a}$ Coresponding author: pierre.casoli@cea.fr
}

This is an Open Access article distributed under the terms of the Creative Commons Attribution License 2.0, which permits unrestricted use, distribution, and reproduction in any medium, provided the original work is properly cited. 


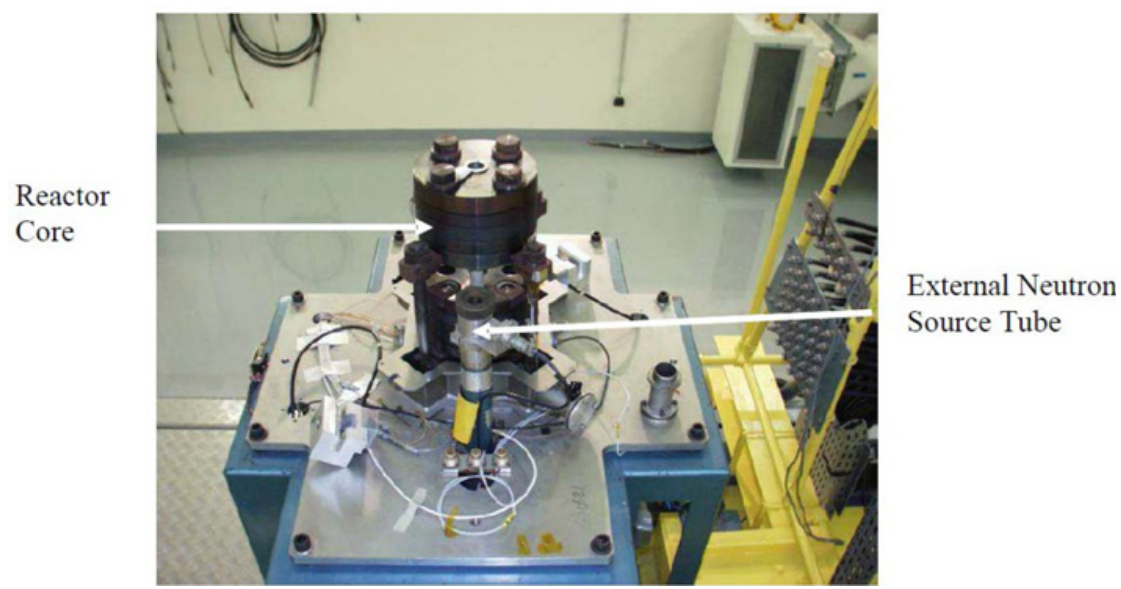

Figure 1. View of CALIBAN reactor without its boron carbide hood.

First, MAXED and GRAVEL, two codes from the UMG package [2] developed by the German PTB were evaluated and results obtained with this software were compared with calculation from the neutron transport code TRIPOLI-4 [3] and from the activation code FISPACT [4].

More recently, CALMAR [5], a new adjustment code currently under development at the CEA Center of Cadarache, was tested to study CALIBAN cavity neutron spectrum.

\section{CALIBAN Critical Assembly}

\subsection{Reactor Description}

CALIBAN is a metallic core cylindrical reactor, vertically oriented (see Fig. 1). Its diameter is $19.5 \mathrm{~cm}$ and its height is $25 \mathrm{~cm}$. It is composed of two blocks: a fixed upper block and a movable lower block. Each block is composed of five metallic plates. The latter are made of uranium highly enriched in uranium 235 (93.5\%) alloyed with $10 \%$ in weight of molybdenum. Four cylindrical control rods, made of the same alloy as the blocks, allow operating the reactor.

The whole core is covered by a steel and boron carbide hood, which limits the effect on the core of the neutrons backscattered from the cell walls. The reactor includes a central irradiation cavity situated inside the two blocks. An external Pu-Be neutron source can be placed in a dedicated tube close to the reactor, and is used during the reactor starting step.

\subsection{Reactor Working Modes and Performances}

The CALIBAN reactor is mainly operated in two modes: a steady state power mode and a pulsed mode.

The pulsed mode is the most used mode. The process is divided in four steps (see Fig. 2):

1. A reactivity calibration of the reactor, using the external neutron source, allows the determination of the critical position of the control rods. After about 45 minutes, the reactor is stabilized at a critical state with a power of about $30 \mathrm{~mW}$.

2. A small reactivity step is added by a slight additional insertion of one of the control rods. The reactor diverges until a power of about $300 \mathrm{~mW}$. The introduced reactivity worth is calculated from the measured divergence period. 


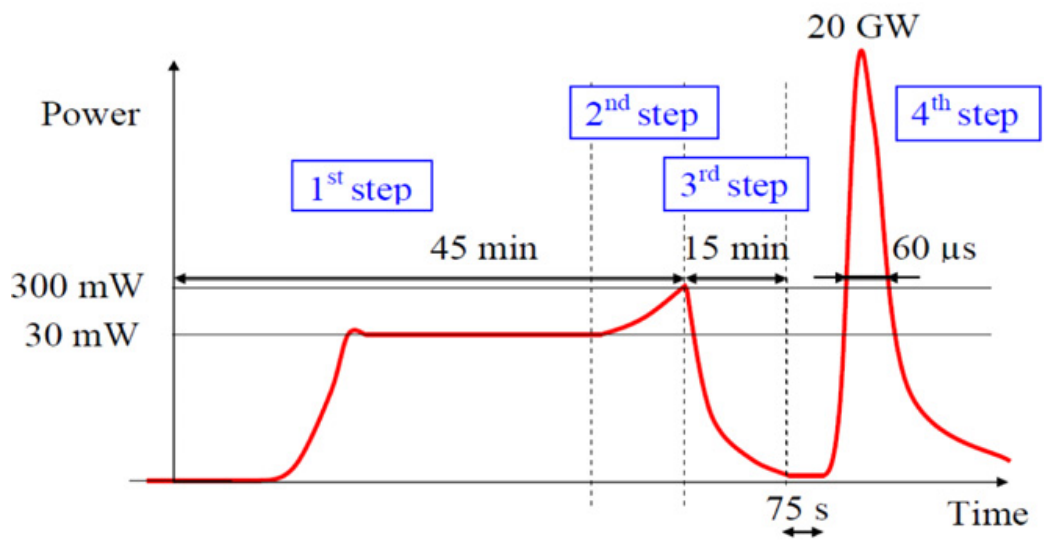

Figure 2. CALIBAN pulsed mode process.

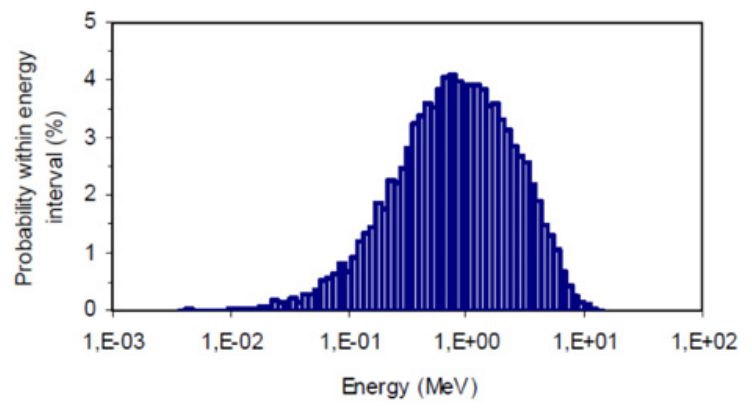

Figure 3. Neutron spectrum in CALIBAN irradiation cavity, TRIPOLI-4 simulation.

3. The lower mobile bloc is moved down. The neutron population in core decreases for 15 minutes to its lower level.

4. The mobile bloc is quickly moved in contact with the fixed one and the excursion rod is pushed in the core. When a persistent neutron chain occurs, the neutron population increases exponentially in the core: the power pulse starts. The core heats up and dilates. This dilatation has two consequences. First, it induces an anti-reactivity which stops the power increase. Then the mobile bloc pushes on the fixed one and falls down: the reactor becomes strongly subcritical back.

In the steady state mode, the maximum power for CALIBAN is $600 \mathrm{~W}$, i.e. $2 \times 10^{13}$ fissions/s, with a limitation of $3.6 \times 10^{17}$ fissions, i.e. 5 hours at maximal power. At this maximal power, the neutron flux is about $1.2 \times 10^{11} \mathrm{n} / \mathrm{cm}^{2} / \mathrm{s}$ in the cavity and about $1.2 \times 10^{10} \mathrm{n} / \mathrm{cm}^{2} / \mathrm{s}$ outside of the core, one centimeter from the hood.

In the pulsed mode, CALIBAN reaches a superprompt critical state: the pulse maximum power is about $20 \mathrm{GW}$ for a corresponding FWHM of $60 \mu \mathrm{s}$. In this mode, the neutron fluence is about $4 \times 10^{14} \mathrm{n} / \mathrm{cm}^{2}$ in the irradiation cavity and the maximum neutron flux at the top of the pulse is about $4 \times 10^{18} \mathrm{n} / \mathrm{cm}^{2} / \mathrm{s}$ in the irradiation cavity.

Neutron spectrum in CALIBAN cavity is very close to fission spectrum. Calculations performed with the TRIPOLI-4 [3] simulation code have shown that the cavity neutron mean energy is about $1.35 \mathrm{MeV}$. Therefore this reactor is particularly suitable to integral experiments requiring a fission spectrum and high neutron flux (see Fig. 3). 


\section{Scientific Studies Completed using CALIBAN}

\subsection{Radiation Effects}

Initially designed for hardening studies, CALIBAN is still extensively used for these kinds of experiments, providing neutron and gamma mixed fluxes on samples and devices to be studied. For these irradiations, CALIBAN is operated either in the steady state mode, or in the pulsed mode, following effects to be studied.

Especially, CALIBAN is used for electronic hardening studies, and silicon displacement damage testing analyses are completed using the reactor. For these experimental programs, the fluences equivalent to $1 \mathrm{MeV}$ in silicon received by the components during the irradiations have to be evaluated, therefore the neutron spectrum provided by the core needs to be known with a high accuracy.

\subsection{Nuclear Data Evaluation}

The benchmark of CALIBAN provides an accurate description of the reactor, which allows the modelling of experiments carried out using the core and the analysis of achieved experimental results.

Especially, this benchmark has been used recently to design and analyse experimental programs devoted to integral measurements carried out to test evaluated nuclear databases.

As a first example, experiments performed using CALIBAN in the seventies and early eighties provided measurements of many fission product yields for fission neutron induced reactions on uranium 235, uranium 233 and plutonium 239. The results of these experiments, analyzed and published recently for the first time [6, 7], have been compared with evaluated data and are in good agreement for reactions on uranium 233 and uranium 235. Nevertheless the experimental results seem to underestimate some evaluations, especially for reactions on plutonium 239. To explain these discrepancies and also improve further the accuracy of the results of these experiments, a new fission product yields measurement program is currently under progress using CALIBAN.

As another example, reactivity worth experimental measurements by the perturbation method have been carried out for several years using CALIBAN and especially in the framework of a recent $\mathrm{PhD}$ [8]. These experiments consist in putting a small sample in the irradiation cavity of the reactor and measuring its effect on the reactivity of the core. Many elements and isotopes, fissile or not, have been studied: gold, lutetium, uranium 238, plutonium $239 \ldots$ In this experimental program, three reactivity measurements techniques, suitable for the CALIBAN reactor, were implemented: replacement measurements, divergence asymptotic period measurements and rod drop experiments.

Once again, a good knowledge of the neutron spectra provided by the core is essential to the analysis of the results of each of these experimental programs.

\section{Activation Foils Measurements}

\subsection{Nuclear Data Evaluation}

The Radiation Metrology Laboratory of the Criticality, Neutron Science and Measurement Department allows the measurement of activation foils used for neutron flux evaluation for experiments using CALIBAN.

The laboratory uses the following types of measurement equipment:

- three gamma spectrometry devices equipped with N-type semiconductor germanium detectors (HPGe). These detectors work on energy fields ranging from $60 \mathrm{keV}$ to $2 \mathrm{MeV}$. The minimum detectable activity is about few tenths of Becquerel for samples with long halflives; 


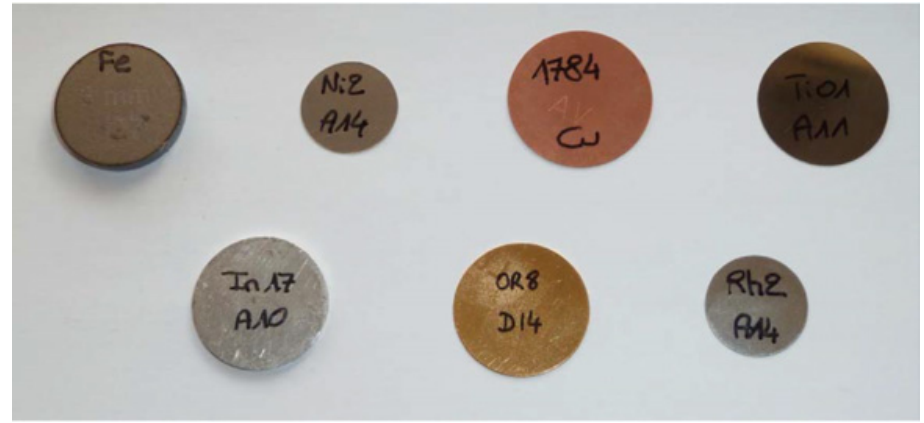

Figure 4. Set of sensors of elements used for CALMAR adjustment.

- one gamma spectrometry device equipped with a broad-energy germanium detector and dedicated to low level gamma spectrometry. This detector is built with materials selected to generate low background.

A Plexiglas device located on the detector allows the placement of the sample at several vertical heights ranging from contact to a maximum of $10 \mathrm{~cm}$, with high accuracy and repeatability.

All the detectors are placed in shielding castles with graded liners of high purity copper with a thickness of $2 \mathrm{~mm}$. One of the castles is an ultra-low background shield, made up of a series of several layers (low and ultra low background lead, cadmium, copper, and Perspex).

The efficiency calibrations of the gamma spectrometry devices are calculated with the CANBERRA software LABSOCS. Several software tools are used to evaluate correction factors for self-absorption, geometry, and peak sums. For intense photo-peak and low background measurements, the total uncertainty of activity measurements is about $1.5 \%(1 \sigma)$.

The Radiation Metrology Laboratory uses also fission chamber detectors specially designed for radiation measurements and thermo-luminescent gamma ray dosimeters.

In 2013, the Radiation Metrology Laboratory took part in the EWGRD Round Robin measurement exercise [9].

\subsection{Activation Foils List and Activation Measurement Results}

The activation foils used for neutron flux evaluation are called sensors or dosimeters. They are disks with thicknesses ranging from $125 \mu \mathrm{m}$ to $2 \mathrm{~mm}$ and diameters ranging from 2 to $22 \mathrm{~mm}$. They are made of high purity materials (at least $99 \%$ pure).

Figure 4 displays a set of activation foils corresponding to the activation reactions used for the spectrum adjustments obtained with the CALMAR software and presented further in this article.

Sensors of these elements were placed in the past in the CALIBAN central cavity and measured activation reaction rates are given in the following Table 1.

\section{Neutron Spectra Adjustments}

\subsection{UMG Adjustments}

UMG is a software developed by the German PTB Research Center. It is composed of two programs, MAXED and GRAVEL, which can be used to provide a 'best fit' of a neutron flux spectrum from a set of activation foil measurements. 
Table 1. CALIBAN sensor activities.

\begin{tabular}{|c|c|c|c|c|}
\hline Sensors & Reactions & $\begin{array}{c}\text { Activation } \\
\text { Products }\end{array}$ & $\begin{array}{c}\text { Measured reaction } \\
\text { rates }\left(\mathbf{1 0}^{-\mathbf{2 4}} \mathbf{s}^{-\mathbf{1}}\right)\end{array}$ & $\begin{array}{c}\text { Measured } \\
\text { uncertainties }(\boldsymbol{\%}, \mathbf{1} \sigma)\end{array}$ \\
\hline Iron & ${ }^{54} \mathrm{Fe}(\mathrm{n}, \mathrm{p})^{54} \mathrm{Mn}$ & ${ }^{54} \mathrm{Mn}$ & $2.32 \times 10^{7}$ & 3 \\
\hline Nickel & ${ }^{58} \mathrm{Ni}(\mathrm{n}, \mathrm{p})^{5} \mathrm{Co}$ & ${ }^{5} \mathrm{Co}$ & $3.27 \times 10^{7}$ & 3 \\
\hline Copper & ${ }^{63} \mathrm{Cu}(\mathrm{n}, \alpha)^{60} \mathrm{Co}$ & ${ }^{60} \mathrm{Co}$ & $1.47 \times 10^{5}$ & 3 \\
\hline Titanium & ${ }^{46} \mathrm{Ti}(\mathrm{n}, \mathrm{p})^{46} \mathrm{Sc}$ & ${ }^{46} \mathrm{Sc}$ & $3.10 \times 10^{6}$ & 3 \\
\hline Indium & ${ }^{115} \mathrm{In}\left(\mathrm{n}, \mathrm{n}^{\prime}\right)^{115 \mathrm{~m}} \mathrm{In}$ & ${ }^{115 \mathrm{~m}} \mathrm{In}$ & $6.57 \times 10^{7}$ & 3 \\
\hline Gold & ${ }^{197} \mathrm{Au}(\mathrm{n}, 2 \mathrm{n})^{196} \mathrm{Au}$ & ${ }^{196} \mathrm{Au}$ & $1.04 \times 10^{6}$ & 5 \\
\hline Rhodium & ${ }^{103} \mathrm{Rh}\left(\mathrm{n}, \mathrm{n}^{\prime}\right)^{103 \mathrm{~m}} \mathrm{Rh}$ & ${ }^{103 \mathrm{~m}} \mathrm{Rh}$ & $2.92 \times 10^{8}$ & \\
\hline
\end{tabular}
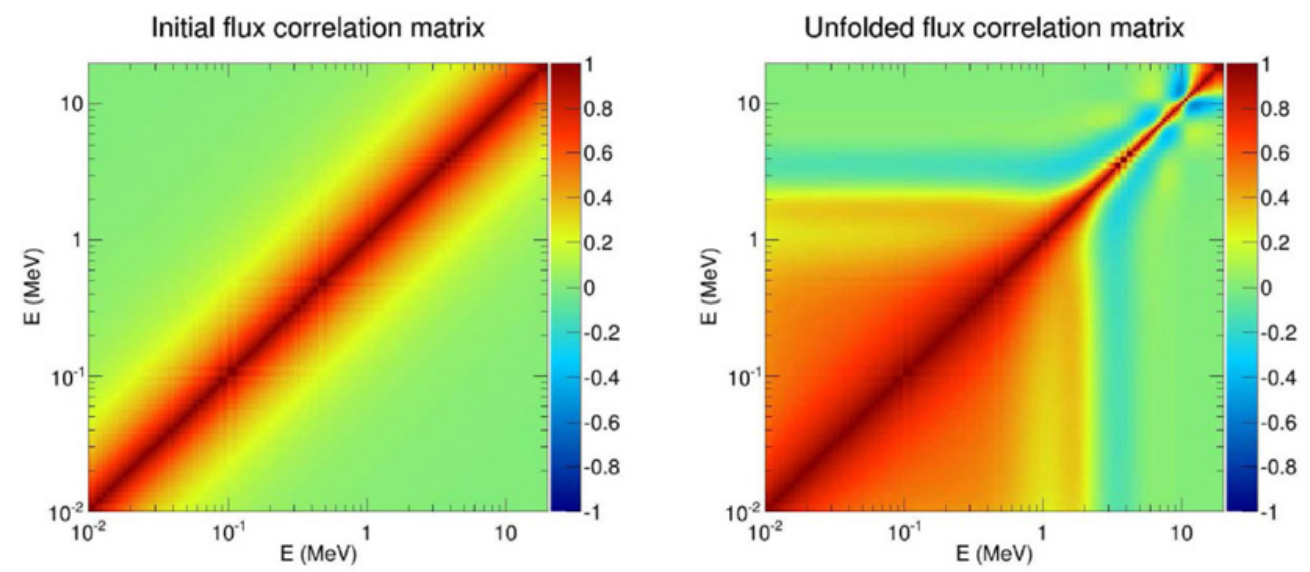

Figure 5. Correlation matrices computed by CALMAR, for CALIBAN cavity neutron spectrum, using a set of seven activation sensors.

The MAXED program is based on the maximum entropy principle. The solution is obtained by the maximization of the entropy of the system, taking into account constraints imposed by the measurements. MAXED needs a prior spectrum as input data.

The GRAVEL program is based on an iterative least square method, using a modified SANDII algorithm. A prior spectrum is needed as input data. It is modified at each calculation step so that the solution fits better the activation measurements.

MAXED and GRAVEL have been applied on a set of activation foil and fission chamber measurements carried out in the CALIBAN. GRAVEL convergence is good even with a flat prior spectrum, while MAXED needs a prior spectrum with finer information, as a TRIPOLI-4 spectrum, to give a correct result [4].

\subsection{CALMAR Adjustments}

CALMAR is a program currently under development at the CEA Center of Cadarache. As the GRAVEL program, it allows the unfolding of a neutron spectrum from a set of activation measurements, using an iterative least square method, but in addition it can take into account information given in a correlation matrix related to the prior spectrum. The prior spectrum can be generated by a Monte-Carlo neutron transport code, such as TRIPOLI-4, and tools have been developed to build the related correlation matrix. CALMAR can also take into account nuclear data uncertainties.

In 2012, CALMAR was applied to a set of activation foil measurements but the convergence of the unfolding was not satisfying and some improvements in the code were needed [10]. 


\section{$15^{\text {th }}$ ISRD}

Table 2. CALIBAN sensor experimental activities compared to UMG and CALMAR evaluations.

\begin{tabular}{|c|c|c|c|c|}
\hline \multirow{2}{*}{ Reactions } & $\begin{array}{c}\text { Experimental } \\
\text { reaction rates }(\mathbf{E}) \\
\left(10^{-24} \mathbf{s}^{-\mathbf{1}}\right)\end{array}$ & \multicolumn{3}{|c|}{ Computed reaction rates $(\mathbf{C})\left(10^{-24} \mathbf{s}^{\mathbf{- 1}}\right)$ and C/E values } \\
\cline { 3 - 5 } & & $\begin{array}{c}\text { MAXED } \\
\text { (from 17 sensors) }\end{array}$ & $\begin{array}{c}\text { GRAVEL } \\
\text { (from 17 sensors) }\end{array}$ & $\begin{array}{c}\text { CALMAR } \\
\text { (from 7 sensors) }\end{array}$ \\
\hline${ }^{54} \mathrm{Fe}(\mathrm{n}, \mathrm{p})^{54} \mathrm{Mn}$ & $2.32 \times 10^{7}$ & $2.49 \times 10^{7}(1.07)$ & $2.50 \times 10^{7}(1.08)$ & $2.33 \times 10^{7}(1.01)$ \\
\hline${ }^{58} \mathrm{Ni}(\mathrm{n}, \mathrm{p})^{58} \mathrm{Co}$ & $3.27 \times 10^{7}$ & $3.35 \times 10^{7}(1.02)$ & $3.38 \times 10^{7}(1.07)$ & $3.27 \times 10^{7}(1.00)$ \\
\hline${ }^{63} \mathrm{Cu}(\mathrm{n}, \mathrm{a})^{60} \mathrm{Co}$ & $147 \times 10^{5}$ & $157 \times 10^{5}(1.07)$ & $143 \times 10^{5}(0.97)$ & $1.66 \times 10^{5}(113)$ \\
\hline${ }^{46} \mathrm{Ti}(\mathrm{n}, \mathrm{p})^{46} \mathrm{Sc}$ & $3.10 \times 10^{6}$ & $341 \times 10^{6}(110)$ & $321 \times 10^{6}(1.04)$ & $332 \times 10^{6}(1.07)$ \\
\hline${ }^{115} \mathrm{In}\left(\mathrm{n}, \mathrm{n}^{\prime}\right)^{115 \mathrm{~m}} \mathrm{In}$ & $6.57 \times 10^{7}$ & $6.52 \times 10^{7}(0.99)$ & $6.58 \times 10^{7}(\mathrm{I} .00)$ & $6.55 \times 10^{7}(1.00)$ \\
\hline${ }^{197} \mathrm{Au}(\mathrm{n}, 211)^{196} \mathrm{Au}$ & $1.04 \times 10^{6}$ & $1.09 \times 10^{6}(1.09)$ & $1.02 \times 10^{6}(0.98)$ & $1.13 \times 10^{6}(1.08)$ \\
\hline${ }^{103} \mathrm{Rh}\left(\mathrm{n}, \mathrm{n}^{\prime}\right)^{1033} \mathrm{Rh}$ & $2.92 \times 10^{8}$ & $2.86 \times 10^{8}(0.98)$ & $2.85 \times 10^{8}(0.98)$ & $2.86 \times 10^{8}(0.98)$ \\
\hline
\end{tabular}

CALMAR was then upgraded and the algorithm of the code was modified to compute simultaneously level and shape adjustments [5]. In comparison with STAYSL, a least square adjustment code included in CALMAR package, the level adjustment adds new correlations between the energy groups, whereas the shape adjustment lowers the correlations.

This new version of the code has been applied to the set of activation foils measurements given in Table 1, using the IRDFF 1.03 nuclear database. The prior spectrum was calculated by TRIPOLI-4, using the JEFF3.1 nuclear database, following a 150 energy group structure ranging from $10 \mathrm{keV}$ to $20 \mathrm{MeV}$. The correlation initial matrices were built using the ASTM E721 data. Figure 5 displays the correlation added by CALMAR between the initial and the unfolded flux correlation matrices.

Activation reactions rate for each foil of the set have been computed with CALMAR and are compared with the results given by MAXED and GRAVEL in Table 2. CALMAR adjustment results seem to be at least as good as UMG results. Additional studies, especially about the effect of the number and of the type of sensors included in the adjustment set, would be needed for further analysis.

\section{Conclusion}

Work is still in progress to improve the adjustments of CALIBAN spectrum using the recently developed version of CALMAR. First results shows that the new code takes into account the activation measurements to build the flux correlation matrices more efficiently than the previous one and that computed reaction rates are at least as good as the ones computed with UMG codes. Additional studies have to be completed to evaluate for example the effect of the sensor set selection or the energy group structure on the unfolding process. Nevertheless, it appears that a benchmarked reactor as CALIBAN is a tool particularly suitable for this kind of unfolding software testing.

\section{References}

[1] N. Authier et al., Bare Highly Enriched Uranium Fast Burst Reactor CALIBAN, HEU-METFAST080, ICSBEP Handbook, NEA Nuclear Science Committee (2007)

[2] M. Reginatto, The Few Channel Unfolding Programs in the UMG Package, UMG Software Instructions (2004)

[3] O. Petit et al., TRIPOLI-4 Version 4 User Guide, CEA-R-6169 Technical Report (2008)

[4] X. Jacquet et al., Journal of ASTM International, Vol. 9, No. 3, JAI104020 (2012)

[5] G. Grégoire et al, CALMAR: a new versatile code library for adjustment from measurements, Proceedings of 15th ISRD, (This proceeding)

[6] J. Laurec et al., Nuclear Data Sheets 111, 2965 (2010) 
[7] P. Casoli et al., Journal of ASTM International, Vol. 9, No. 4, JAI104018 (2012)

[8] B. Richard, Mesures de perturbations sur le réacteur CALIBAN - Interprétation en terme de qualification de données nucléaires, PhD thesis, University of Paris-Sud (2013)

[9] D.A. Thornton et al, The EWGRD Round Robin Measurement Exercise, Proceedings of $15^{\text {th }}$ ISRD, (To be published)

[10] P. Casoli et al, Characterization of the CALIBAN and PROSPERO Critical Assemblies Neutron Spectrums for Integral Measurements Experiments, Proceedings of the International Conference on Nuclear Data for Science and Technology, New York (USA) 2013, Nuclear data Sheets 118 (2014), p. 554-557 